
\title{
25 Research Square \\ Fluid Status Changes During the Transition in Infants of Diabetic Mothers.
}

\author{
Vincenzo Zanardo ( $\nabla$ vincenzo.zanardo@libero.it ) \\ Abano Polyclinic: Policlinico Abano \\ Domenico Tortora \\ Istituto Giannina Gaslini \\ matteo Parotto \\ $\mathrm{U}$ of T: University of Toronto \\ Valeria Manghina \\ Abano Polyclinic: Policlinico Abano \\ lorenzo Severino \\ Abano Polyclinic: Policlinico Abano \\ Pietro Guerrini \\ Abano Polyclinic: Policlinico Abano \\ Gianluca Straface \\ Abano Polyclinic: Policlinico Abano
}

\section{Research Article}

Keywords: Fluid status at birth, Infants of diabetic mothers, Transition, Wet lung

Posted Date: April 19th, 2021

DOl: https://doi.org/10.21203/rs.3.rs-407561/v1

License: (c) (i) This work is licensed under a Creative Commons Attribution 4.0 International License.

Read Full License

Version of Record: A version of this preprint was published at European Journal of Pediatrics on July 15th, 2021. See the published version at https://doi.org/10.1007/s00431-021-04197-7. 


\section{Abstract}

\section{Background}

In animal and human neonates, expansion of the extracellular fluid volume is associated with "wet" lung and poor respiratory outcomes.

\section{Methods}

To define fluid status changes during the transition from fetal to neonatal life in infants of diabetic mothers (IDM), we conducted a single centre (Policlinico Abano Terme, Abano Terme, Italy) study of 66 IDM and a 1:2 matched control group from January 1 to September 30, 2020. Fluid status changes were assessed by computing $\Delta \mathrm{Hct}$ from umbilical cord blood at birth and capillary heel Hct at $48 \mathrm{~h}$, accounting for body weight decrease.

Results

IDM presented with significantly lower cord blood Hct levels in comparison to controls $(47.33 \pm 4.52 \mathrm{vs}$ $50.03 \pm 3.51 \%, p<0.001)$, mainly if delivered by elective cesarean section $(45.01 \pm 3.77$ vs $48.43 \pm 3.50 \%$, $p=0.001)$. Hct levels at $48 \mathrm{~h}$ were comparable $(55.18 \pm 5.42$ vs $54.62 \pm 7.41 \%, p=0.703)$, concurrently with similar body weight decrease $(-217.21 \pm 113.34 \mathrm{vs}-217.51 \pm 67.28 \mathrm{~g}, \mathrm{p}=0.614)$. This supports significantly higher $\Delta$ Hct in IDM $(5.13 \pm 5.24$ vs $7.29 \pm 6.48, p<0.01)$ and extra circulating fluid loss of $2-3 \%$.

Conclusion

Gestational diabetes is associated with an excess of circulating fluids during the transition from fetal to neonatal life, challenging the current assumption that is per se at risk of wet lung.

\section{What Is Known}

In neonates, evidence suggests that expansion of the extracellular fluid volume prior to the postnatal diuresis is associated with poor respiratory outcomes.

\section{What Is New}

Gestational diabetes is associated with an excess of circulating fluids during the transition from fetal to neonatal life, challenging the current assumption that is per se at risk of wet lung.

\section{Introduction}

The body composition of the fetus changes during gestation with a smaller proportion of body weight composed of water as gestation progresses [1]. Non-labor uterine contractions during the last few days before delivery and stronger uterine contractions or mild hypoxia during labor and delivery result in even 
greater reduction in fetal blood volumes and hence plasma [2]. In animal and human neonates, evidence suggests that expansion of the extracellular fluid volume prior to the postnatal diuresis is associated with poor neonatal respiratory outcomes, as an essential part of the rapid change from the "wet" lung of the fetus to the "dry" lung needed by the neonate for gas exchange [3,4]. Fetal lung fluid, which is in essence amniotic fluid, contains very little protein and has a low colloid osmotic pressure. It is removed by Increased pulmonary perfusion, which pulls lung fluid from the alveoli across the capillary membrane [5]. Therefore, it seems clinically relevant to consider that the pulmonary function in premature neonates requiring mechanical ventilation deteriorates until the onset of diuresis, after which it rapidly improves [6]. This diuresis may represent the removal of excess lung liquid and seems necessary for improvement in lung function. Evidence suggests that particularly infants delivered prematurely or by elective caesarean delivery and neonates of diabetic mothers are prone to respiratory problems at birth, most often manifested as transient tachypnea of the newborn (TTN) and respiratory distress syndrome (RDS) [7]. It is also reassuring that postnatal physiological weight loss is largely due to the removal of excess lung liquid and seems necessary for improvement in RDS [6].

Although these data make a valuable contribution to the growing body of knowledge surrounding prelabour and perinatal fluid changes, clinical studies have not differentiate determinants and effects of fetal fluid status changes in infants of gestational diabetes mothers (IDM), one of the single most important factors affecting placental function, fluid status changes of the fetus and newborn and finally neonatal pulmonary function at birth. This is relevant, considering that in a nationwide study in Sweden of a large series of pregnancies complicated by gestational diabetes, perinatal mortality rate was not increased; but the rate of transient tachypnea and fetal asphyxia were two to three times higher than normal [8].

Therefore, there is a clear need to define the various levels relative total body water and extracellular fluid excess in the offspring of diabetic mothers that may have an adverse lung effect. Of equal importance is to standardize and systematize the criteria used to assess the significance of any such impact. The main objective of this study was, hence, to assess fluid status in healthy neonates of gestational diabetic mothers. The main hypothesis stated that $\Delta \mathrm{Hct}$ at 48 hours of age, generated starting from cord blood Hct and capillary heal Hct levels, accounting for postnatal physiological body weight decrease, would be better representative of fluid status changes [9] in infants delivered by a gestation uneventful or gestational diabetes complicated condition.

\section{Patients And Methods}

This is a case-control (1:2 ratio) study conducted at Policlinico Abano Terme in Abano Terme, Italy from January to September 2020. The hospital where the study took place is located in an industrialized area of North-Eastern Italy supporting advanced educational levels, good socio-economic status, occupation, and low and late fertility in about 1000 births per year. The Data on all neonates of gestational diabetes mothers were prospectively collected following a study protocol approved by the Scientific Committee for the Policlinico Abano Terme (SC-PAT), Abano Terme, Italy. The SC-PAT deemed this study to be of 
minimal risk with no interaction and no intervention with human subjects and thus, was exempt from consent. The data include prenatal demographic details, maternal pregnancy history and antenatal care, details of the delivery, the infant's status at delivery, diagnoses, procedures and complications during hospitalization, and outcome at discharge.

A total of 656 at term infants ( $>37<42$ gestational weeks) were born at the Division of Perinatal Medicine during the 9-month study period, from January 1 to September 30, 2020. From the cohort we identified 66 (10.02\%) infants of gestational diabetes mothers (IDM), which composed our study group. A control group composed of 2 infants for each case, matched for parity, gestational age ( \pm 1 week) and delivery mode, was selected on the basis of the first compatible live-born infant before and after each study infant.

All women who presented at term for an elective caesarean section or underwent unplanned emergency caesarean section were screened for eligibility. The eligibility criteria included singleton low-risk pregnancy, gestational age of $>37-<42$ weeks confirmed by a first trimester ultrasound, and screening of gestational diabetes at 24-28 week given by the woman.

Exclusion criteria included maternal medical and obstetric major complications. After delivery, the infant was excluded if there was a confirmed diagnosis of intrauterine growth restriction or congenital anomalies or NICU admission, presence of Rh or major ABO isoimmunization indexed by a positive direct antiglobulin test, and drugs administered to the infants, except for 1 mg vitamin $\mathrm{K}$ (Konakion ${ }^{\circledR}$ ) intramuscularly soon after birth. Neonates were also excluded if an umbilical artery blood sample for blood gas analysis and Hct levels were not or were incorrectly obtained.

The study was carried out within the context of the hospital's ordinary care protocol. Mothers who underwent elective cesarean delivery (EICD) were fasting at least 6 hours before the caesarean section and by delivery room protocol received parenteral Ringer lactate solution prior to the loco-regional anesthesia procedure. In all cases after surgical birth, according to our local protocol, we used intravenous oxytocin routinely after caesarean delivery, and at the same time, the umbilical cord was clamped. Infants from either group were handed to the awaiting pediatric staff and moved to an infant warmer. Following an uneventful surgical delivery, in accordance with the hospital's standard practice, infants were right after placed on the mother's chest for about 15 minutes during which time the midwife assisted with the first suckling episode. During the subsequent 2 days in our ward, newborns roomed-in with their mothers, who were encouraged to demand feed them (with no more than 3-hour interfeeding intervals). The infants received complementary formula milk if breast milk intake was judged insufficient by the midwives.

There were two data collection points: at birth and at 48 hours of age (time of hospital discharge). At birth, resuscitative measures, birth weight, blood gas analysis and Hct were recorded. Umbilical artery blood gas analysis and complementary tests were measured by ABL90 FLEX Radiometer ${ }^{\circledR}$ analyzer $\left(\right.$ Radiometer $^{\circledR}$ ). At 48 hours of age, to avoid unnecessary discomfort for the newborn, a capillary heel ct 
was drawn simultaneously with the routine pre-discharge blood work for the mandatory neonatal metabolic screenings. The following infant data were also collected from the hospital birth record: birth weight, physiological body weight decrease, and percentage of weight loss, polycythemia (Hct $>65 \%$ ) or anemia $(\mathrm{Hct}<47 \%)[10]$, bilirubinemia, and any undesired neonatal conditions.

Statistical analysis. Patient characteristics and outcome measure were summarized with descriptive purpose using mean and standard deviation (continuous data) or frequencies and percentages (categorical data). Assessment of the magnitude of fluid status changes was performed by computing $\Delta$ Hct from umbilical cord blood at birth and capillary heel Hct at 48 hours, which provides a crude assessment of fluid shift from the intravascular compartment, accounting for the physiological body weight decrease in the first 48 hours of life. A power analysis with a 1.40 effect size, two-tailed test, an alpha of 0.001 and a power of 0.90 revealed that a sample size of 59 neonates would be sufficient to test the study's primary outcome. Comparisons of clinical and laboratory blood parameters were performed between IDM and controls using a general linear model analysis. The statistical threshold for rejecting the null hypothesis was set at $P<0.05$ adjusted applying a Bonferroni correction for multiple correction. Statistical analysis was performed by using SPSS Statistics for Mac, version 21.0 (IBM).

\section{Results}

Two IDM were excluded from the analysis (1 with TTN and 1 with incomplete hemo gas analysis). Accordingly, data of the remaining 64 infants were considered for the statistical analysis. Among GD pregnant women, all were treated with diet and 3 with diet in association with insulin. Mothers presented with low (Nulliparous, $62(48.44 \%)$ vs 31 (48.44\%) and late (Years, $35.18 \pm 4.75$ vs $35.75 \pm 6.06, p=0.44$ ) fertility and high cesarean delivery rates, elective [22 (34.38\%) vs $44(34.38 \%), p=1.00]$ and emergency [14 (10.94\%) vs 7 (10.94\%), $p=1.00]$.

In particular, at birth, IDM and control neonates presented comparable arterial cord blood gas analysis and substantially concurrent values, except for potassium, higher in the control group $(p=0.047)$. Table 1

Differently, at birth, IDMs presented with significantly higher glycemia levels than the control group (84.77 \pm 19.37 vs $78.3 \pm 19.16 \mathrm{mg} \%, \mathrm{p}=0.007)$ and the difference resulted higher in IDM delivered in the absence of labor $(68.43 \pm 9.55$ vs $61.46 \pm 8.38 \mathrm{mg} \%, p=0.004)$. Figure 1

Conversely, IDM presented with significantly lower Hct levels in comparison to control group (47.33 \pm 4.52 vs $50.04 \pm 3.51 \%, p<0.001)$ and the difference resulted significantly higher in IDM delivered in the absence of labor $(45.01 \pm 3.77$ vs $48.42 \pm 3.50 \%, p=0.001)$. Figure 2

At the same time, more IDMs presented subclinical anemia (Hct 47\%) in arterial cord blood at birth $(27,42.19 \%$ vs $24,18,75 \%, p<0.001)$, and none presented policytemia (Hct>65\%). At discharge, on the second day of life, IDMs and control group presented with comparable capillary Hct levels $(55.18+5.42$ vs $54.62+7.41 \%, p=0.703)$. In fact, $\Delta$ Hct of IDM significantly increased $(5.13 \pm 5.24$ vs $7.29 \pm 6.48 \%, p<0.01)$, 
despite a comparable physiological weight loss decrease. $(-217.21+113.31$ vs $-217.51+67.28 \mathrm{~g}, \mathrm{p}=0.614)$. At the same time, bilirubin levels were comparable between groups. Table 1

\section{Discussion}

In this study, it was found, for the first time, that IDM present a dilutional anemia in arterial cord blood at birth, suggestive of excess circulating fluids during the transition from fetal to neonatal life. Conversely, at 48 hours of postnatal life, IDM showed similar Hct level of control group, having restored their hematological status by a significant $\Delta$ Hct increase, mirroring an extra circulating plasma loss by $2 \%$ to $3 \%$, in presence of a comparable physiological weight loss decrease.

Current analysis of knowledge related to neonatal transition reveals that excess circulating fluids or expansion of the extracellular fluid volume may interfere with completion of a normal physiologic transition [11]. An end result of this interference with transitional physiology is that particularly infants delivered prematurely or at term by elective caesarean delivery, deprived of the eustress of being born, are prone to respiratory problems at birth, most often manifested as need for neonatal resuscitation at birth, air leak, TTN, and RDS [12]. Furthermore, in the near term and term neonate excess fluid administration results in generalized edema and abnormalities of pulmonary function [13].

The mechanisms that mediate fluid status changes and dilutional anemia in the fetal to neonatal establishment of respiratory autonomy are complex and far from completely understood. The transition from fetal to neonatal life is associated with what have come to be accepted as normal changes in fluid balance. A physiological decrease in extracellular water volume, as well as an increase in packed red cell volume must be taken into account as it mirrors the amount of fluids excess [9]. In general, the more immature the newborn, the greater changes can be expected, mainly if delivered by elective cesarean section, deprived of plasma catecholamine, vasopressin or cortisol concentrations $[9,14]$. As background, it is important to recognize two facts. First, it is blood volume and not plasma volume that is regulated in the fetus and neonate, just as it occurs in adults [15]. Second, one of the major determinants of plasma loss across the body capillaries is the combined effects of hydrostatic and osmotic pressure gradients at the capillary membrane [16]. The lymphatic system counterbalances this egress by pumping interstitial fluid through the lymphatic vessels back in the circulation [16]. Thus, in the steady state of IDM the balance between plasma and interstitial fluid, seems possibly determined, in presence of substantially comparable cord blood electrolytes and hemo gas analysis values, by the relationship between osmotic pressure changes induced by transient hyperglycemia, mainly in the presence of labor. Several animal studies collectively suggest that pre-labor mild uterine contractions have a direct compression or conformational change on the fetus and may elevate (20\%) vascular pressure during the last few days before delivery, reducing circulating blood volume by $2 \%-4 \%$ in fetal lambs [17]. Thus, stronger uterine contractions during labor would be expected to result in even greater reduction in fetal blood volumes and hence plasma volumes [18]. In this scenario, the lungs of fetal lambs begin to dry out several days before delivery, in large part because the amount of fluid secreted by the lungs gradually decreases as a result of changes in plasma catecholamine, vasopressin or cortisol concentrations [19]. Furthermore, mild hypoxia 
reduces circulating fetal volume, which results from a loss of plasma volume into the interstitial space and exert a major effect on the transcapillary distribution of fluid due to the associated elevations in arterial and venous pressures [17]. An end result of this interference with transitional physiology is that particularly infants delivered prematurely or at term by elective caesarean delivery, deprived of the eustress of being born, are prone to respiratory problems at birth, most often manifested as TTN and RDS [12]. Care of the IDMs needs to focus on ensuring adequate fluid balance and diuresis in cardiorespiratory adaptation at birth, lying in a state of relative total body water and extracellular fluid excess, mainly if delivered early by elective cesarean section.

These data may have some clinical relevance. In particular, these findings may challenge the current assumption that IDM are per se at risk of wet lung and even more severe respiratory problems at birth. Human babies are remarkably adaptable, and in most cases no apparent harm is initially evident. However, it is important to establish a rationale for early neonatal fluid excess and management and then to apply it in clinical practice. Nevertheless, $\Delta$ Hct increases at 48 hours of life may result in a crude formula useful to define and compare excess fluid status severity and changes in different gestational and delivery settings supporting a wet lung in a wet baby. This seems relevant, considering that despite $\mathrm{L} / \mathrm{S}$ ratios indicating fetal lung maturity, respiratory distress continues to occur more frequently in infants of diabetic mothers [20]. Moreover, qualitative chromatographic assays of surfactant phosphatides revealed that non-insulin-dependent diabetic pregnancies had lowest mean per cent phosphatidylglycerol [21].

Although these data make a valuable contribution to the growing body of evidence surrounding pre-labor and perinatal fluid changes in IDM, they are limited by the impossibility to extend these conclusions to all deliveries (i.e., preterm and near-term gestations with and without prenatal steroid treatment to induce lung maturity...). Moreover, we were unable to assess the contributing role of other placental mechanisms classically associated with fetal expansion of the extracellular fluid volume (i.e., major isoimmunizations, chorioangioma, fetal anemia...) and associated with poor neonatal respiratory outcomes. Finally, the limitations associated with these measurements in IDM must be taken into account in interpreting the results, controlling for $\Delta \mathrm{Hct}$ from birth to 48 hours of life, with a crude clinical formula, accounting for postnatal body weight decrease, but irrespective of diuresis and relative large and high variable insensible water loss determinations [22].

In conclusion, our data show that IDM present an excess of circulating fluids during the transition from fetal to neonatal life, by the contributing effect of transient hyperglycemia, that enhanced osmotic pressure and reduces capillary filtration in utero and challenges the current assumption that IDM are per se at risk of respiratory problems at birth. Subsequently, at 48 hours of life, IDM restore their hematological status by a significant $\Delta$ Hct increase.

\section{Abbreviations}

IDM, infants of gestational diabetes mothers. 
TTN, tachypnea of the newborn.

RDS, respiratory distress syndrome.

\section{Declarations}

Funding: No financial support for this research.

Conflicts of interest: The authors disclose any conflict of interest.

Availability of data and material: The data supporting our findings can be found in the Archives of the Policlinico Abano Terme, stored in electronic format.

Code availability: N/A

Authors' contributions: VZ carried out the study. DT and MP participated in study design. VM and LS participated in study design and coordination. PG and GS helped draft the manuscript. All authors read and approved the final manuscript.

Ethics approval: Study protocol approved by the Scientific Committee for the Policlinico Abano Terme (SC-PAT), Abano Terme, Italy.

Consent to participate: The Scientific Committee for the Policlinico Abano Terme deemed this study to be of minimal risk with no interaction and no intervention with human subjects and thus, was exempt from consent.

Consent for publication: The Scientific Committee for the Policlinico Abano Terme consented the publication.

\section{References}

1. Toro-Ramos T, Paley C, Pi-Sunyer FX, Gallagher D (2015) Body composition during fetal development and infancy through the age of 5 years. Eur $J$ Clin Nutr 69:1279-289

2. Turner JM,Murray DM, Kumar SS (2020)The physiology of intrapartum fetal compromise at term. Am J Obstet Gynecol 222:17-26

3. Barker PM , Olver E R (2002) Lung Edema Clearance: 20 Years of Progress. Invited Review: Clearance of lung liquid during the perinatal period. J Appl Physiol 93: 1542-1548

4. O'Brien F, Walker IA (2014) Fluid homeostasis in the neonate. Paediatr Anaesth24:49-59

5. Levick JR, Michel CC (2010) Microvascular fluid exchange and the revised Starling principle. Cardiovasc Res 15; 87:198-210

6. Heaf DP, JBelik HJ, Spitzer AR, Gewitz MH, Fox WW (1982) Changes in pulmonary function during the diuretic phase of respiratory distress syndrome. J Pediatr 10:103-107 
7. Lucky J, Dudell GD (2006) Respiratory transition in infants delivered by cesarean section. Semin Perinatol 30:296-304

8. Fadl HE, Ostlund IKM, Magnuson AFK, Hanson USB (2010)Maternal and neonatal outcomes and time trends of gestational diabetes mellitus in Sweden from 1991 to 2003. Diabet Med27:436-441

9. Zanardo V, Tortora D, Parotto M, Severino L, Simbi A, Guerrini P, Straface G (2020) Determinants and effects of fluid status changes in caesarean delivered neonates. Acta Paediatr 1091:545-550

10. Luchtman-Jones L, Schwartz AL, Wilson DB (2006) Hematologic problems in the fetus and neonate. In: Martin RJ, Fanaroff AA, Walsh C, eds. Fanaroff and Martins Neonatal-Perinatal Medicine. Diseases of the Fetus and Infant, 8th edn. Mosby-Elsevier, Philadelphia, PN, pp. 1287-1356

11. Hillman N, Kallapur SG, Jobe A (2012) Physiology of Transition from Intrauterine to Extrauterine Life. Clin Perinatol 39:769-783

12. Lagercrantz $H$ (2016) The good stress of being born. Acta Paediat 2:1413-416

13. Lucky J, Eaton DC (2006) Physiology of Fetal Lung Fluid Clearance and the Effect of Labor. Semin Perinatol 30:34-43

14. Young A, Brown LK, Enmis S, Beattie M, Johnson MJ (2021) Total body water in full-term and preterm newborns: systematic review and meta-analysis. Archives of Disease in Childhood - Fetal and Neonatal Edition Published Online First: 31 March 2021. doi: 10.1136/archdischild-2020-321112

15. Brace RA 1983() Blood volume and its measurement in the chronically catheterized sheep fetus. Am J Pysiol 244:H487-H94

16. Brace RA, Gold PS (1984) Fetal whole-body interstitial compliance, vascular compliance, and capillary filtration coefficient. Am J Physiol 247:R800-R5

17. Brace RA (1996) Blood volume responses to acute fetal hypoxia. Am J Obstet Gynecol I155:889.

18. Brace RA (2004) Fluid distribution in the fetus and neonate. In: Polin RA, Fox WW (ed)) Fetal and Neonatal Physiology. Saunders, New York1341-1350

19. Pfister RE, Ramsden CA, Neil HL, Kyriakides MA, Berger PJ (2001) Volume and secretion rate of lung liquid in the final days of gestation and labour in the fetal sheep. J Physiol15:889-99

20. Bourbon JR, Farrell P M (1985) Fetal Lung Development in the Diabetic Pregnancy. Pediatr Res 19:253-267

21. Cunningham MD, Desai NS, S, Thompson A, Greene JM (1978) Amniotic fluid phosphatidylglycerol in diabetic pregnancies. Am J Obstet Gynecol131:719-724

22. Lorenz JM (1977) Assessing fluid and electrolyte status in the newborn. National Academy of Clinical Biochemistry. Clin Chem43:205-210

\section{Tables}

Table 1. Characteristics of the infants of diabetic mothers and control groups (ratio 1:2). 


\begin{tabular}{|c|c|c|c|}
\hline Mean \pm SD or $N(\%)$ & CONTROL & IDM & $p$ \\
\hline Infants: & 128 & 64 & \\
\hline \multicolumn{4}{|l|}{ Arterial cord blood at birth: } \\
\hline $\mathrm{pH}$ & $7.33 \pm 0.07$ & $7.32 \pm 0.07$ & 0.149 \\
\hline $\mathrm{PaO}_{2}, \mathrm{mmHg}$ & $25.72 \pm 9.01$ & $24.82 \pm 9.02$ & 0.256 \\
\hline $\mathrm{PaCO}_{2}, \mathrm{mmHg}$ & $43.19 \pm 9.14$ & $44.33 \pm 9.65$ & 0.203 \\
\hline $\mathrm{HCO}_{3}{ }^{-}, \mathrm{mmol} / \mathrm{L}$ & $21.17 \pm 2.25$ & $20,79 \pm 2.45$ & 0.188 \\
\hline $\mathrm{ABE}, \mathrm{mmol} / \mathrm{L}$ & $-2.74 \pm 2.85$ & $-3.24 \pm 3.23$ & 0.268 \\
\hline $\mathrm{Na}^{+}, \mathrm{mmol} / \mathrm{L}$ & $134.42 \pm 1.93$ & $134.64 \pm 2.18$ & 0.222 \\
\hline $\mathrm{K}^{+}, \mathrm{mmol} / \mathrm{L}$ & $4.74+0.81$ & $4,51 \pm 0.62$ & 0.047 \\
\hline $\mathrm{Cl}^{-}, \mathrm{mmol} / \mathrm{L}$ & $1.41 \pm 0.07$ & $1,42 \pm 0.06$ & 0.396 \\
\hline Lactate, $\mathrm{mmol} / \mathrm{L}$ & $3.08 \pm 1.86$ & $3.27 \pm 2.19$ & 0.404 \\
\hline Glycemia, mg/dL & $78.3 \pm 19.16$ & $84.77 \pm 19.37$ & 0.007 \\
\hline Hct, \% & $50.04 \pm 3.51$ & $47.33 \pm 4.52$ & $<0.001$ \\
\hline Gestational age, w & $39.12 \pm 1.15$ & $38.94 \pm 1.17$ & 0.172 \\
\hline Neonatal birth weight, g & $3277.28 \pm 421.16$ & $3300.15 \pm 399.25$ & 0,462 \\
\hline \multicolumn{4}{|l|}{ At discharge: } \\
\hline Body weight, g & $3060.08 \pm 398.03$ & $3082.66 \pm 382.62$ & 0.519 \\
\hline Weight loss, g & $-217.21 \pm 113.34$ & $-217.51 \pm 67.28$ & 0.614 \\
\hline Hct, \% & $55.18 \pm 5.42$ & $54.62 \pm 7.41$ & 0.703 \\
\hline$\Delta \mathrm{Hct}$ & $5.14 \pm 5.24$ & $7.29 \pm 6.48$ & 0.01 \\
\hline Bilirubin, mg/dL \% & $7.74 \pm 2.37$ & $8.23 \pm 2.86$ & 0.539 \\
\hline
\end{tabular}

Notes: IDM, Infants of diabetic mothers. P indicates the Bonferroni-corrected statistical significance of the general linear model analysis comparing IDM and controls.

\section{Figures}




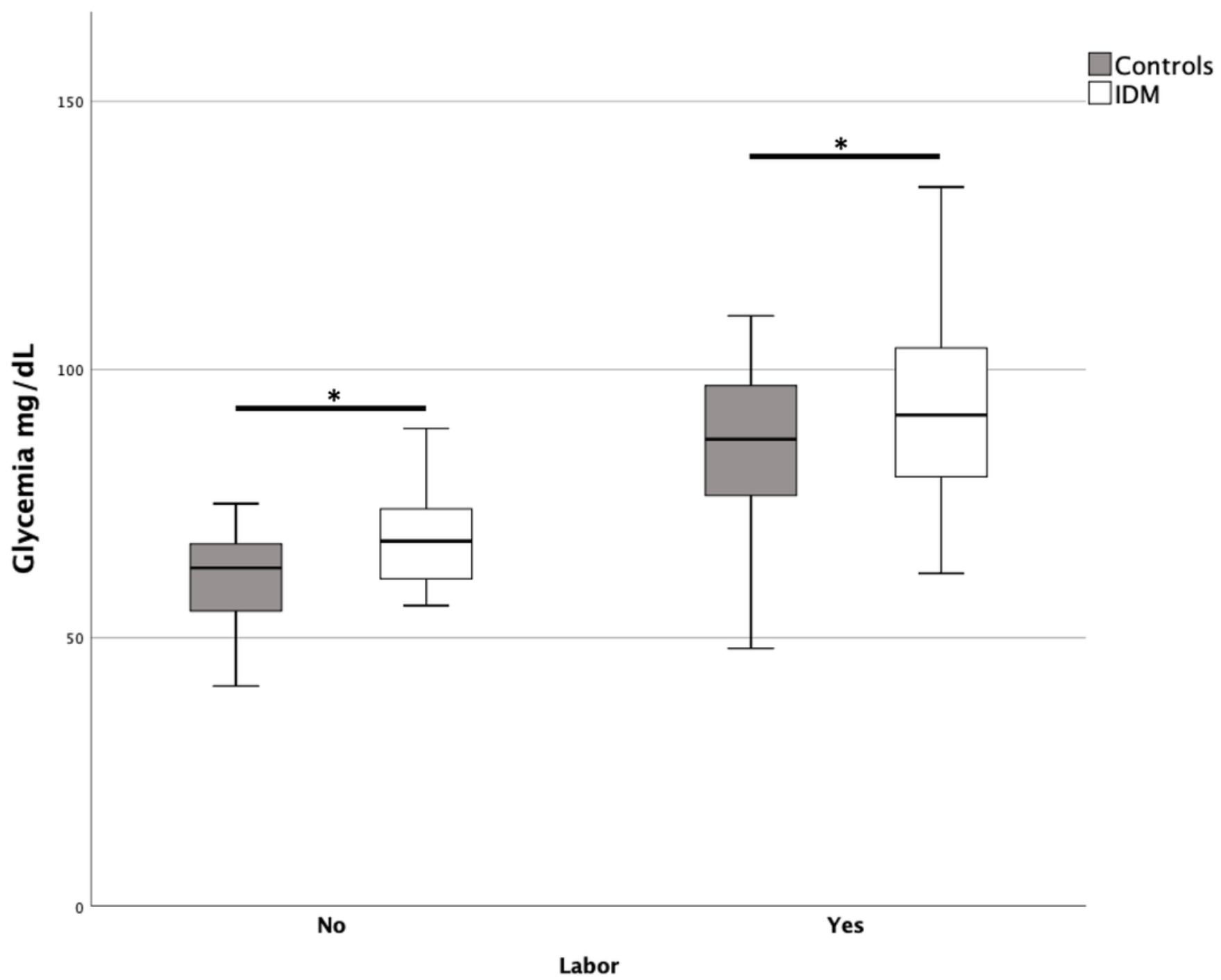

Figure 1

Glycemia levels in arterial cord blood at birth in infants of diabetic mothers and in the control group, in presence and absence of labor. Note: Comparisons between IDMs and controls were performed using a general linear model analysis; * indicates $\mathrm{P}<0.05$. 


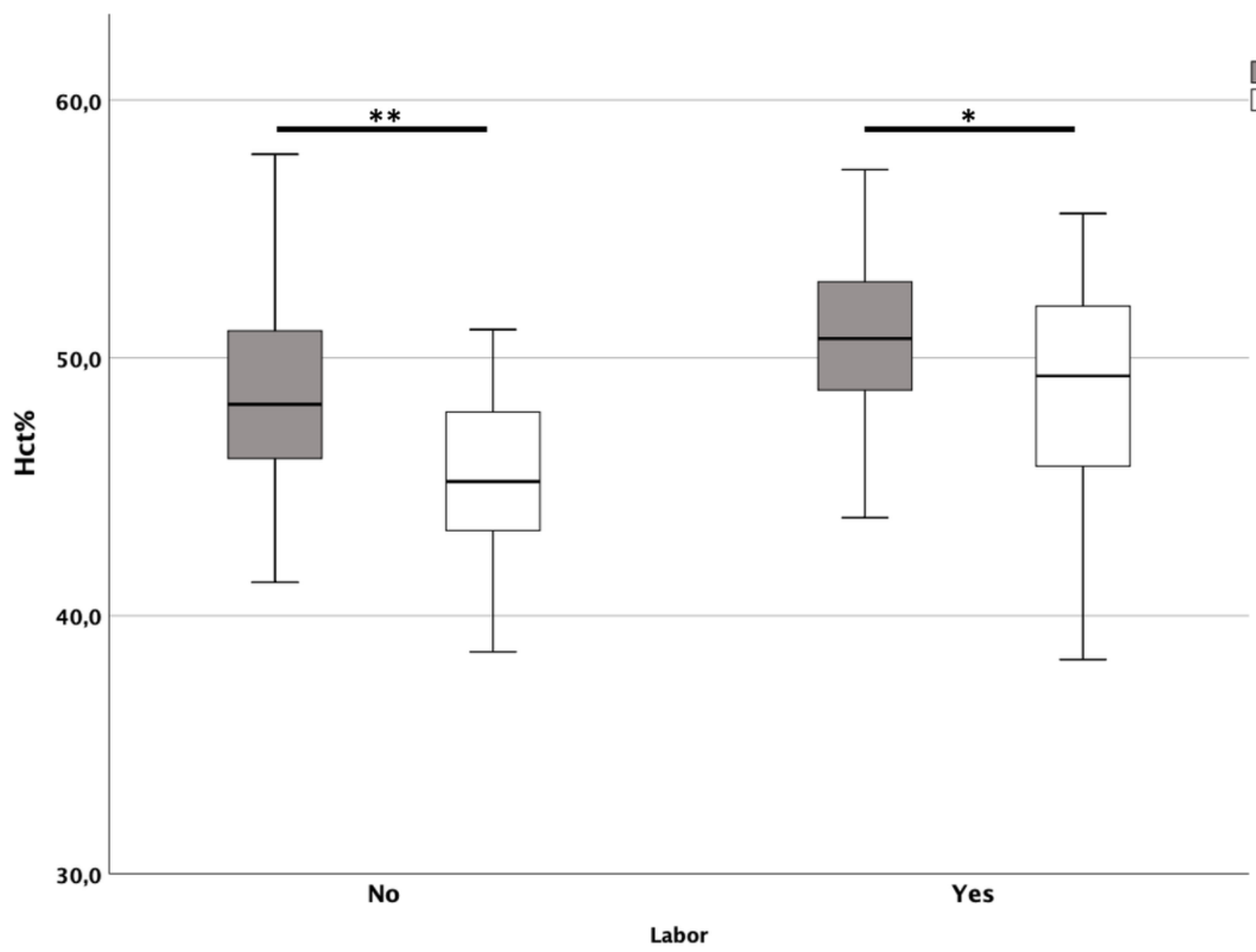

Figure 2

Hematocrit levels in arterial cord blood at birth in infants of diabetic mothers and in the control group, in presence and absence of labor. Note: Comparisons between IDMs and controls were performed using a general linear model analysis; ** indicates $\mathrm{P} \leq 0.001$; * indicates $\mathrm{P}<0.05$. 